\title{
Es geht doch!
}

Es geht doch auch ohne embryonale Stammzellen! Und es geht sogar mittels Herzkatheter! Die Düsseldorfer Arbeitsgruppe um Strauer hat eindrucksvoll demonstriert, dass am infarktgeschädigten Herzen eine Gewebeneogenese offensichtlich unter Verwendung der körpereigenen Stammzellen aus dem Knochenmark des betroffenen Patienten nach intrakoronarer Applikation möglich ist.

Es geht also auch beim Menschen und nicht nur bei Mäusen, dass ein schwer geschädigter linker Ventrikel neue Kraft entwickelt aus den eigenen pluripotenten Zellen, die zu neuem Herzgewebe erwachsen. Ähnliche Resultate wurden für den intramyokardialen Einsatz von gezüchteten peripheren Skelettmuskelzellen bisher am Menschen erzielt.

Die Ergebnisse der Untersuchungen von Strauer und Mitarbeitern (vgl. dieses Heft, S. 932) zeigen erstmals, dass eine Infarkttherapie am Menschen mit der dargestellten PTCA-Technik möglich und klinisch erfolgreich sein kann. Die autologe Stammzelltransplantation mittels selektiver Herzkathetertechnik kann zur Regeneration der Narbe nach transmuralem Myokardinfarkt führen. Ursächlich hierfür dürfte eine Kardiomyo- und Angiogenese sein.

Die Neo-Perfusion und Vitalitätsverbesserung in der vorher avitalen Infarktzone hat bei dem behandelten 46-jährigen Patienten zu einer erheblichen Abnahme der Infarktgröße und zu einer deutlichen Verbesserung der Ventrikelfunktion und der Geometrie des Herzens in Ruhe und unter Belastung geführt.

Es funktioniert also mit körpereigenen Stammzellen, die nach entsprechender Präparation und Anreicherung mittels eines neuen Katheterverfahrens im Infarktgebiet und seinen Randzonen deponiert werden. Die dargestellte Niedrig-DruckPTCA mit temporärer Okklusion des Infarktgefäßes und fraktionierter Überdruckinjektion der Stammzellsuspension ermöglicht die Deponierung in das betroffene Gewebe, ohne dass ein herzchirurgisches Vorgehen mit Thorakotomie und intramyokardialer Zellinjektion oder eine transkoronare bzw. transmyokardiale Injektionstechnik erforderlich ist.

In der kardiologischen Forschung ist nun ein weiterer sehr großer Schritt getan. Natürlich sind noch viele Fragen offen, die beantwortet werden und klinische Studien initiieren müssen, zum Beispiel:

- Wieviele Zellen sind zur Transplantation überhaupt nötig?

- Kann die Geweberegeneration durch Wachstumsfaktoren weiter verbessert werden?

- bildet sich aus den Stammzellen wirklich nur das Gewebe, das benötigt wird?

- Ist eine "Vorbehandlung" der autologen Stammzellen vor Transplantation sinnvoll, z.B. mit endothelialen Wachstumsfaktoren?

- Welche Rolle spielt das Alter der Patienten?
- Ist nach Transplantation »enhanced environment» (starke körperliche oder pharmakologische Belastung) angezeigt, oder eher Ruhigstellung?

Welch phantastische Möglichkeiten eröffnen sich für die Kardiologie, aber auch für andere Gebiete, wenn nun besonders aufbereitete körpereigene Stammzellen mittels Katheterverfahren zur Therapie in die betroffenen Organe appliziert werden können. Dies dürfte erst der Auftakt einer grundlegend neuen Therapieentwicklung sein. Eine vor wenigen Jahren noch als utopisch betrachtete Behandlung eines defekten Organs ist durch einen intelligenten Ansatz mit einer klar konzipierten Vorgehensweise, hier der intrakoronaren autologen Stammzelltransplantation erstmalig erfolgreich durchgeführt worden.

Realisiert wurde das durch eine vorbildliche interdisziplinäre Zusammenarbeit. Es geht also auch bei uns mit der klinischen Forschung auf höchstem Niveau! Es ist uns allen zu wünschen, dass die konsequente Fortentwicklung dieser Technik nicht nur in der Kardiologie die Diskussion um die embryonale Stammzellforschung in einem weiten Bereich erübrigt. So werden erfolgreiche Alternativen aufgezeigt. Die Versprechungen von skrupellosen Embryonenforschern, die ohne klares klinisches Ziel klonen und experimentieren sind inzwischen unerträglich. Bemerkenswerterweise haben gerade die Amerikaner mit ihrem Verbot nun ein klares Zeichen gegeben. Die biotechnologische Revolution kann auch ohne embryonale Stammzellen stattfinden, wenn die Alternativen entwickelt werden.

Da diese Daten klinisch äußerst relevant, hochinnovativ und aktuell sind, war natürlich auch eine rasche Publikation angezeigt. Die DMW freut sich, diese Arbeit innerhalb von 28 Tagen nach Eingang einschließlich "peer review" und Revision veröffentlichen zu können. Wir verbinden damit die Hoffnung, dass dank der konsequenten qualitativen Fortentwicklung unserer Zeitschrift in den letzten Jahren, die DMW an "alte» Zeiten anknüpfen kann und Pionierarbeiten der klinischen Forschung aus dem deutschsprachigen Raum wieder hier veröffentlicht werden. Entgegen dem allgemeinen Trend deutschsprachiger Medizinzeitschriften ist übrigens auch der Impact factor der DMW in den letzten Jahren wieder kontinuierlich angestiegen. Es geht doch auch noch auf Deutsch! Der volle Text der Arbeit ist natürlich auch in englischer Sprache im Internet (http://www.thieme-connect.de) nachzulesen. Ein weiterer Zugang führt über http://www.thieme.de/dmw. Dort haben wir eine Direktverbindung von der Startseite auf den Beitrag eingerichtet.

Prof. Dr. Martin Middeke,

Chefredakteur der DMW 